\title{
First-line Treatment of Advanced Biliary Ducts Carcinoma: A Randomized Phase II Study Evaluating 5-FU/LV Plus Oxaliplatin (Folfox 4) Versus 5-FU/LV (de Gramont Regimen)
}

\author{
GIOVANNI SCHINZARI ${ }^{1}$, ERNESTO ROSSI ${ }^{1}$, GIUSEPPINA MAMBELLA ${ }^{2}$, \\ ANTONIA STRIPPOLI ${ }^{1}$, RODOLFO CANGIANO ${ }^{3}$, MASSIMILIANO MUTIGNANI ${ }^{4}$, \\ MICHELE BASSO $^{1}$, ALESSANDRA CASSANO $^{1}$ and CARLO BARONE ${ }^{1}$ \\ ${ }^{1}$ Medical Oncology, Università Cattolica del Sacro Cuore, Rome, Italy; \\ ${ }^{2}$ Medical Oncology, IRCCS CROB, Rionero in Vulture, Potenza, Italy; \\ ${ }^{3}$ Medical Oncology, Presidio Ospedaliero S. Felice a Cancello, Caserta, Italy; \\ ${ }^{4}$ Surgical and Interventional Endoscopy, Ospedale Niguarda, Milan, Italy
}

\begin{abstract}
Background/Aim: Few clinical trials are available for advanced biliary tract carcinoma (BTC). We conducted this randomized phase II clinical trial to explore efficacy and safety of 5-fluorouracillleucovorin (5-FU/LV de Gramont) or the same regimen plus oxaliplatin (Folfox 4) as first-line treatment of advanced BTC. Patients and Methods: Primary endpoint was overall survival (OS); secondary endpoints were progression-free survival (PFS), response and toxicity. Results: A total of 48 patients were enrolled, 23 in de Gramont arm and 25 in the Folfox arm. Disease control rate was $56.5 \%$ for de Gramont vs. $72 \%$ for Folfox. RR was $21.7 \%$ for de Gramont arm and $28 \%$ for Folfox arm ( $p=0.12)$. PFS was in favor of Folfox (5.2 vs. 2.8 months; $p=0.031$ ). OS was 7.5 and 13.0 months for de Gramont and Folfox arm respectively $(p=0.0010)$. Toxicity was generally mild in both arms. Conclusion: Folfox 4 could be considered a valid option as first-line treatment of BTC due to its efficacy and tolerability.
\end{abstract}

Cancer of the biliary system is a relative rare, but very aggressive neoplasm. It includes cancer of extra- or intrahepatic biliary ducts, cancer of gallbladder and cancer of Vater ampulla. It accounts for $3 \%$ of all gastrointestinal cancers worldwide with slight male predominance (1). The

This article is freely accessible online.

Correspondence to: Giovanni Schinzari, Medical Oncology, Università Cattolica del Sacro Cuore, L.go F. Vito, 1 00168, Rome, Italy. Tel: +390630156318, e-mail: giovanni.schinzari@unicatt.it

Key Words: Advanced biliary duct carcinoma, 5-FU/LV, oxaliplatin, Folfox 4, de Gramont regimen. mainstay of treatment and the only curative approach is radical surgery. Unfortunately, cancer of biliary tract is often diagnosed in metastatic or locally advanced stage. In this condition prognosis is dismal, with less than $5 \%$ of patients alive after 5 years and a median survival less than 1 year (2). Due to the lack of larger randomized phase III trials, for many years no standard chemotherapy regimen in advanced disease was established. In addition, phase II studies are often small and include patients with pancreatic cancer or hepatocellular carcinoma. These studies have identified some active agents: 5-fluorouracil (5-FU), gemcitabine (Gem), cisplatin (Cis) and oxaliplatin (Ox) demonstrated a moderate activity, with a response rate (RR) ranging from $8 \%$ to about $50 \%$ (in monotherapy and in combination) and an overall survival (OS) ranging from 6 to 16 months (3). Based on the efficacy in gastro-intestinal cancer, 5-FU was the first agent investigated in advanced biliary system cancer. Falkson (4) obtained a RR less than $10 \%$ and an OS of 21 weeks in gallbladder cancer and 26 weeks in bile duct cancer with oral 5-FU. Glimelius (5) conducted the first randomized study, in which the combination of 5-FU plus leucovorin (LV) +/Etoposide was compared with best supportive care (BSC) in 36 pancreatic or bile ducts cancer patients. OS was significantly superior in the treatment arm (6 months versus 2.5 months); no difference was observed in the etoposide arm versus 5-FU/LV alone arm. Gem was used in monotherapy by Metzger (6) in 13 patients: 1 partial response and 11 disease stabilization were reported, while OS was 16 months. Other authors $(7,8)$ explored Gem in monotherapy, with a RR of about $20 \%$ and OS of 6.3-11.5 months. A small number of subsequent phase II studies analyzed the efficacy of Gem in combination with other agents. Among these, Gebbia (9) found a RR of $36 \%$ and an OS of 11 months with Gem plus 5-FU. The first randomized 
phase III trial by Valle (10) (ABC-02) demonstrated a significant longer progression-free survival (8 months vs. 5 months) and overall survival (11.7 months vs. 8.1 months) of Gem+Cis vs. Gem alone. Grade 3-4 hematological toxicity was $32.3 \%$ in the Cis+Gem arm and $23.6 \%$ in Gem arm; grade 3-4 fatigue was observed in $18.7 \%$ of patients in the combination arm.

Oxaliplatin is a cornerstone drug in the treatment of gastrointestinal carcinomas; its efficacy was evaluated also in cholangiocarcinoma. As single agent (11), Ox demonstrated a modest activity, with an RR of $20.6 \%$ and an OS of 7 months. Ox combined with Gem (12) showed a RR of $35.5 \%$ and a OS of 15.4 months in chemonaïve patients; whereas the RR and the OS observed with Ox plus 5-FU/LV (13) were 56\% and 9.5 months respectively. These studies reported a mild toxicity with grade 3-4 hematological adverse events observed in less than $15 \%$ of patients and grade 3-4 peripheral neuropathy in about $8 \%$ of the population.

Prospective randomized trials investigating the efficacy of Ox plus 5-FU/LV in advanced biliary carcinoma are lacking. Based on the encouraging results of the previous studies, the good toxicity profile and the absence of a standard therapy before the availability of ABC-02 trial, we planned a phase II prospective randomized study to compare the efficacy of Folfox-4 vs. 5-FU/LV. The choice of de Gramont regimen as comparator for our study was supported by the similar activity between 5-FU/LV and Gem in previous phase II studies (3). The activity of fluoropyrimidines in cholangiocarcinoma and gallbladder carcinoma is also supported by recent results in the post-operative treatment. BILCAP (14) trial demonstrated an advantage in OS and relapse free survival for patients treated with capecitabine when compared with patients in the observational arm.

We report the final analysis of efficacy and toxicity related to the comparison between Folfox-4 and de Gramont in advanced BTC.

\section{Patients and Methods}

Previously untreated patients were considered eligible. Other inclusion criteria (Table I) were: measurable disease (RECIST criteria), age $>18$ years, performance status (ECOG) $\leq 2$, adequate renal, liver and bone marrow functions. Presence of any other neoplasm (apart from basocellular carcinoma or cervical cancer) or central nervous system metastases, chronic diarrhea, uncontrolled infections and other serious concurrent illness represented exclusion criteria. Baseline evaluation included medical history, physical examination and tumor assessment by MRI or CT scan of chest, abdomen and pelvis performed within 21 days before study entry. Brain or bone CT/MRI scans were performed if clinically requested.

Written informed consent was required. Eligible patients were randomly assigned in a $1: 1$ ratio to receive intravenously $\mathrm{LV}$ $100 \mathrm{mg} / \mathrm{m}^{2}$ (2 hours infusion) on days 1 and 2, 5-FU $400 \mathrm{mg} / \mathrm{m}^{2}$ (bolus infusion) on days 1 and 2 and 5-FU $1,200 \mathrm{mg} / \mathrm{m}^{2}$ (46 hours infusion) or the same regimen plus oxaliplatin $85 \mathrm{mg} / \mathrm{m}^{2}$ (2 hours infusion) on day 1 . Treatments were administrated every 2 weeks and continued until disease progression or unacceptable toxicity or consent withdrawal. Tumor assessment was performed every 2 months until disease progression.

The primary endpoint of the study was OS, calculated from the day 1 of the first cycle until death. Secondary endpoint was PFS, calculated from the day 1 of the first cycle until progression disease or death, and RR, evaluated according to RECIST criteria. The Kaplan-Meier methods was employed to define time to event outcomes; log-rank test was used for the comparison of survival between arms. A Cox-proportional hazard model was used to calculate the hazard ratios. The differences between RR was evaluated by Pearson $\chi^{2}$ test.

Toxicity was evaluated according to the National Cancer Institute Common Toxicity Criteria (NCI-CTC) version 3. The subsequent cycle was administered if absolute neutrophil count was $>1.5 \times 10^{9} / 1$, platelets count $>100 \times 10^{9} / 1$ and if patient had completely recovered from any other adverse event. Dose reduction (75\% of scheduled dose) was performed in case of recurrent grade 3-4 haematological or non haematological toxicity. Granulocyte-Colony Stimulating Factors (G-CSF) and Erytropoietin (Epo) were employed according to American Society of Clinical Oncology (ASCO) guidelines.

During the enrollment of the study a group of patients fulfilling all inclusion and exclusion criteria refused chemotherapy and underwent symptomatic procedures only, such as biliary stenting. Although they were not randomized, a further analysis was performed considering these patients as a contemporary best supportive care (BSC) group. These patients underwent regular clinical evaluation every 4 weeks until death. Survival was calculated from the day of consent proposal until death. The study has been approved by the local ethics committee.

\section{Results}

From January 2008 to June 201048 patients were enrolled (23 in the de Gramont arm and 25 in the Folfox arm). During the same period 25 patients refused chemotherapy and were included in the BSC group. Patients' characteristics are described in Table II. The median age was 61 years in de Gramont arm and 58 years in Folfox arm. In BSC group the median age was slightly higher (65 years) than in the randomized arms. Biliary decompression by stenting through endoscopic retrograde or percutaneous colangiography was necessary for $48 \%$ of BSC patients and for $22 \%$ of treated patients.

All patients receiving chemotherapy were evaluable for efficacy and toxicity. The median number of delivered cycles was 8 (range=2-18) for Folfox arm and 5 (range=4-11) for de Gramont arm; cumulative dose intensity was $87 \%$ in de Gramont arm and $85 \%$ in Folfox arm. The treatment was discontinued for progressive disease in 46 patients $(95.8 \%)$, while 2 patients in Folfox arm withdrew consent after 11 and 12 cycles of therapy. $45 \%$ of patients in both arms (10 in de Gramont arm and 11 in Folfox 4 arm) had GEM as second line therapy.

Overall response rate was $21.7 \%$ for de Gramont arm and $28 \%$ for Folfox arm ( $p=0.12$ - Table III). Stable disease was 
Table I. Inclusion and exclusion criteria.

Inclusion criteria

Histologically or cytologically confirmed biliary tract adenocarcinoma Misurable Disease (RECIST criteria)

Age $>18$ years old

No previous systemic treatment for advanced disease

Life expectancy of at least 12 weeks

PS (ECOG) $\leq 2$

Serum creatinine $<1.5 \mathrm{UNL}$

Total bilirubine and alkaline phosphatase $<1.5 \mathrm{UNL}$

AST and ALT $<1.2 \mathrm{UNL}$

Absolute neutrophil count $>1.5 \times 10^{9} / 1$

Hemoglobin $>10.0 \mathrm{~g} / \mathrm{dl}$

Platelets $>100 \times 10^{9} / 1$

Written informed consent

Exclusion criteria

Central nervous metastases

History of other neoplasm diagnosed within the last 5 years, except for basocellular carcinoma or cervical cancer

Chronic diarrhoea

History of inflammatory bowel disease

Infections

Any serious concomitant illness

Pregnant or lactating women

PS: Performance status.

Table II. Patients characteristics.

\begin{tabular}{lccc}
\hline & de Gramont & Folfox & BSC \\
\hline Patients & 23 & 25 & 25 \\
Median age (range) & 61.3 & 62 & 65 \\
M/F & $10 / 13$ & $13 / 12$ & $11 / 14$ \\
PS (ECOG) & & & \\
0 & 10 & 9 & 6 \\
1 & 11 & 12 & 10 \\
2 & 2 & 4 & 7 \\
Disease & & & \\
Gallbladder & 6 & 5 & 6 \\
Vater Ampolla & 1 & 2 & 4 \\
$\quad$ Biliary tract & 17 & 18 & 15 \\
Biliary stenting & 4 & 7 & 11 \\
\hline
\end{tabular}

PS: Performance status.

$34.8 \%$ in de Gramont arm and $44 \%$ in Folfox arm. Therefore, disease control rate (DCR) was $56.5 \%$ in patients treated with the de Gramont schedule versus $72 \%$ for those treated with Folfox. No complete response was observed. PFS was 2.8 months in de Gramont arm and 5.2 months in
Table III. Response rate.

\begin{tabular}{lcc}
\hline & $\begin{array}{c}\text { de Gramont } \\
\text { n. }(\%)\end{array}$ & $\begin{array}{c}\text { Folfox } \\
\text { n. }(\%)\end{array}$ \\
\hline Complete response & 0 & 0 \\
Partial response & $5(21.7)$ & $7(28)$ \\
Stable disease & $8(34.8)$ & $11(44)$ \\
Disease control & $13(56.5)$ & $18(72)$ \\
Progression & $10(43.5)$ & $7(28)$ \\
\hline
\end{tabular}

Table IV. Toxicity.

\begin{tabular}{lcc}
\hline & $\begin{array}{c}\text { de Gramont } \\
\text { n. }(\%)\end{array}$ & $\begin{array}{c}\text { Folfox } \\
\mathrm{n} .(\%)\end{array}$ \\
\hline $\begin{array}{l}\text { Haematological (grade III-IV) } \\
\text { Neutropenia }\end{array}$ & $1(4.3)$ & $2(8)$ \\
$\quad \begin{array}{l}\text { Anemia } \\
\text { Thrombocitopenia }\end{array}$ & - & - \\
Gastrointestinal (grade III-IV) & - & - \\
$\quad$ Diarrhoea & $2(8.7)$ & $2(8)$ \\
$\quad$ Mucositis & $3(13.4)$ & $1(4)$ \\
Neuropathy (grade I-II) & $/$ & $3(12)$ \\
Neuropathy (grade III-IV) & $/$ & $1(4)$ \\
\hline
\end{tabular}

Folfox arm [HR 0.4787 (95\%CI=0.2559-0.8952); $p=0.0031$ - Figure 1] while OS was 7.5 and 13.0 months for de Gramont and Folfox arm respectively [HR 0.3121 (95\%CI=0.1535-0.6345) $p=0.0013$ - Figure 2]. No differences in terms of OS and PFS were observed stratifying patients by site of primary tumor. OS in BSC group was 6.6 months.

Toxicity was generally mild and not life-threatening (Table IV). Two patients in the Folfox group and one treated with de Gramont experienced grade III/IV neutropenia. Nonneutropenic fever was observed in only 1 patient in Folfox arm. Grade III diarrhea occurred in 2 patients in de Gramont arm and in 2 patients in Folfox arm, while 3 patients in de Gramont arm and 1 patient in Folfox arm experienced grade III/IV stomatitis. Ox caused sensorial neuropathy in 4 patients $(16 \%)$; among them only one patient reported grade III neuropathy.

\section{Discussion}

The treatment of advanced biliary ducts carcinoma is based only on few clinical trials. The only phase III randomized study available (ABC-02) demonstrated an advantage of $\mathrm{CDDP}+\mathrm{Gem}$ versus Gem alone. This combination regimen is considered a typical treatment for advanced BTC. Data on 


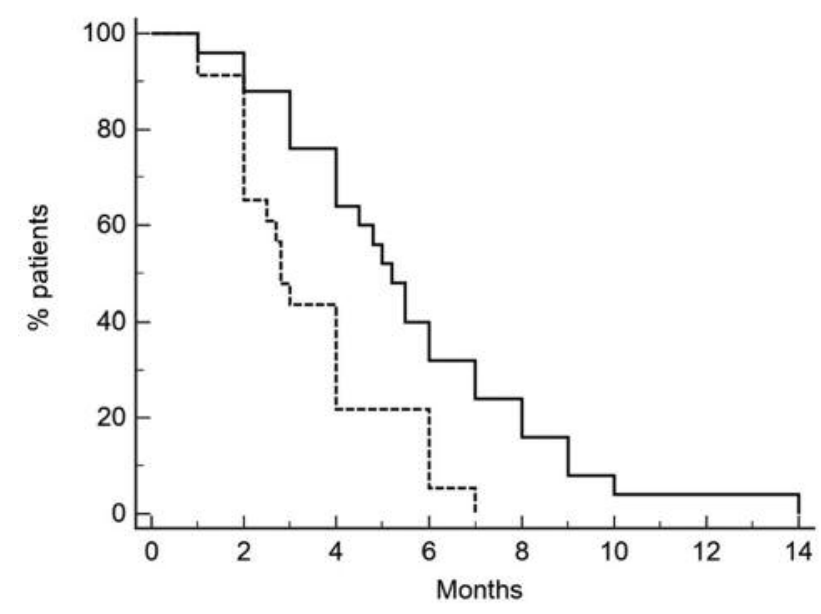

Figure 1. Progression-free survival of Folfox (5.2 months, solid line) vs. de Gramont (2.8 months, dashed line); [HR 0.4787 (95\%CI=0.25590.8952), $p=0.0010]$.

the efficacy and safety of other agents in monotherapy or combination are limited since the relative low incidence of BTC. Before the availability of ABC-O2 trial results, we planned the present study based on the data by previous phase II analyses (13), showing a potential benefit by adding Ox to 5-FU/LV regimen. The efficacy of Folfox-4 in comparison with Gem was also evaluated restrospectively in a more recent study (15), which showed a better outcome for the series of patients treated with the combination regimen (median OS 14.1 months vs. 8.3 months).

In our prospective trial overall survival, the primary endpoint of the study, was clearly superior in patients treated with Folfox-4 in comparison to those receiving 5FU/LV (12.6 months vs. 6.7 months; $p=0.0013)$. Coherently a significant increase in PFS was also observed (5.0 months vs. 2.7 months respectively, $p=0.027$ ). The survival benefit of Folfox- 4 was surprisingly longer than 12 months and similar to $\mathrm{CDDP}+\mathrm{Gem}$ in ABC-02 trial. Furthermore, it does not depend on post study therapies because the percentage of patients exposed to second line treatment was the same in both arms. Another interesting point arising from the present study is the substantially similar OS between 5-FU/LV (6.7) and BSC group (6.1 months). The hypothesis derived from this observation could be the possibility to consider only the BSC for patients not fit for a combination regimen. Modern stenting procedures allow to solve the obstruction of biliary system thus obtaining a palliative control of the disease comparable to that reported with a single agent chemotherapy.

In terms of DCR, we observed also a difference in favor of Folfox-4 versus de Gramont (72\% vs. 56.5\%), similarly

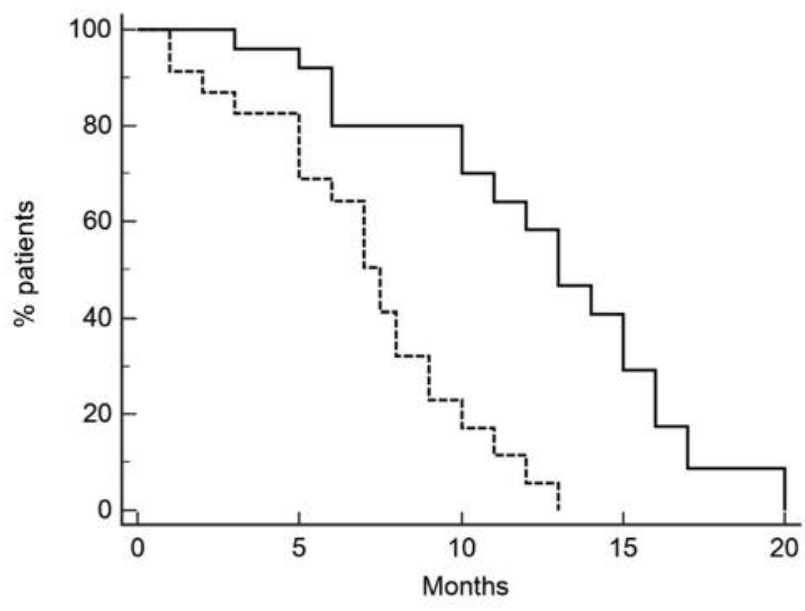

Figure 2. Overall survival of Folfox (13.0 months, solid line) vs. de Gramont (7.5 months, dashed line); [HR 0.3121 (95\%CI=0.15350.6345), $p=0.0031]$.

to combination arm in $\mathrm{ABC}-02$ trial (81.4\% for $\mathrm{CDDP}+\mathrm{Gem}$ vs. $71.8 \%$ for Gem). No difference in RR between Folfox-4 and de Gramont was observed, maybe for the difficulties in assessing the response according to the RECIST criteria in BTC.

In our study, treatments were well tolerated with less than $10 \%$ of patients experiencing grade 3-4 toxicity. Dose intensity was $>80 \%$ in both arms and neither serious adverse events nor toxic deaths were observed. As expected, neurotoxicity complicated Folfox-4 treatment, but only 1 patient reported G3 neuropathy. Overall toxicity profile for Folfox-4 in our study was mild; although the different study design, grade 3-4 toxicity seems less frequent for Folfox-4 than for CDDP-GEM in ABC-02 trial.

In the present study FOLFOX-4 seems to have an activity comparable to $\mathrm{CDDP}+\mathrm{GEM}$ in $\mathrm{ABC}-02$ trial a good tolerability, thus we could consider this combination therapy as a valid option for first line treatment of BTC, especially when patients have been already treated with Gem as postoperative therapy.

Although this study and other trials have shown some benefit by combination therapy, more impressive results for BTC probably could be reached through a better knowledge of the biology of the tumor, with the introduction of effective agents addressing driver molecular alterations.

\section{Conflicts of Interest}

The Authors have no conflicts of interest to declare. 


\section{References}

1 Khan SA, Thomas HC, Davidson BR and Taylor-Robinson SD: Cholangiocarcinoma. Lancet 366: 1303-1314, 2005.

2 Patel T: Worldwide trends in mortality from biliary tract malignancies. BMC Cancer 2: 10, 2002.

3 Hezel AF and Zhu AX: Systemic Therapy for Biliary Tract Cancers. The Oncologist 13: 415-423, 2008.

4 Falkson G, MacIntyre JM and Moertel CG: Eastern cooperative oncology group experience with chemotherapy for inoperable gallbladder and bile duct cancer. Cancer 54: 969-969, 1984.

5 Glimelius B, Hoffman K, Sjodén PO, Jacobsson G, Sellstrom H, Sellstrom H, Enander LK, Enander LK, Linné T and Svensson C: Chemotherapy improves survival and quality of life in advanced pancreatic and biliary cancer. Ann Oncol 7: 593-600, 1996.

6 Metzger J, Sauerbruch T, Ko Y, Wolter H, Funk C and Glasmacher A: A phase II trial of gemcitabine in gallbladder and biliary tract carcinomas. Onkologie 21: 232-234, 1998.

7 Penz M, Kornek GV, Raderer M, Ulrich-Pur H, Fiebiger W, Lenauer A, Depisch D, Krauss G, Schneeweiss B and Scheithauer W: Phase II trial of two-weekly gemcitabine in patients with advanced biliary tract cancer. Ann Oncol 12: 183186,2001

8 Valencak J, Kornek GV, Raderer M, Ulrich-Pur H, Krauss G, Greul R, Pidlich J, Pfeffel F, Haider K, Kwasny W, Schneeweiss $\mathrm{B}$ and Scheithauer W: Gemcitabine for the treatment of advanced biliary tract carcinomas: evaluation of two different dose regimen. Onkologie 22: 498-501, 1999.

9 Gebbia V, Giuliani F, Maiello E, Colucci G, Verderame F, Borsellino N, Mauceri G, Caruso M, Tirrito ML and Valdesi M: Treatment of inoperable and/or metastatic biliare tree carcinomas with single agent gemcitabine or in combintion with levofolin acid and infusional fluorouracil: results of a multicenter phase II study. J Clin Oncol 19: 4089-4091, 2001.
10 Valle J, Wasan H, Palmer DH, Cunningham D, Anthoney A, Maraveyas A, Madhusudan S, Iveson T, Hughes S, Pereira SP, Roughton M, Bridgewater $\mathrm{J}$ and $\mathrm{ABC}-02$ Trial Investigators: Cisplatin plus Gemcitabine versus Gemcitabine for Biliary Tract Cancer. N Eng J Med 362: 1273-1281, 2010.

11 Androulakis N, Aravantinos G, Syrigos K, Polyzos A, Ziras N, Tselepatiotis E, Samonis G, Kentepozidis N, Giassas S, Vamvakas L and Georgoulias V: Oxaliplatin as first line treatment in inoperable biliary tract carcinoma: a multicenter phase II study. Oncology 70: 280-284, 2006.

12 André T, Tournigand C, Rosmorduc O, Provent S, MaindraultGoebel F, Avenin D, Selle F, Paye F, Hannoun L, Houry S, Gayet B, Lotz JP, de Gramont A, Louvet C and GERCOR Group: Gemcitabine combined with oxaliplatin (GEMOX) in advanced biliary tract adenocarcinoma: a GERCOR study. Ann Oncol 15: 1339-1343, 2004.

13 Nehls O, Klump B, Arkenau HT, Hass HG, Greschniok A, Gregor $\mathrm{M}$ and Porschen R: Oxaliplatin, fluorouracil and leucovorin for advanced biliar system adenocarcinoma: a prospective phase II trial. British J Cancer 87: 702-704, 2002.

14 Primrose JN, Fox R, Palmer DH, Prasad R, Mirza D, Anthoney DA, Corrie P, Falk S, Wasan HS, Ross PJ, Wall LR, Wadsley J, T.R. Jeffry Evans, Stocken D, Praseedom R, Cunningham D, Garden OJ, Stubbs C, Valle JW and Bridgewater JA: Adjuvant capecitabine for biliary tract cancer: The BILCAP randomized study. ASCO abstr 4006, 2017.

15 Novarino AM, Satolli MA, Chiappino I, Giacobino A, Napoletano R, Ceccarelli M, Ciccone G, Schena M, Bertetto O and Ciuffreda L: Folfox-4 regimen or single-agent Gemcitabine as first line chemotherapy in advanced biliary tract cancer. Am J Clin Oncol 36: 466-471, 2013.

Received June 14, 2017

Revised July 19, 2017

Accepted July 24, 2017 